Volume 12(2-3) (2015), 117-128

Copyright (c) 2016 Equinox Publishing Ltd

Sheffield

http://equinoxpub.com

DOI: $10.1558 /$ cam.18399

\title{
The relevance of existing health communication models in the email age: An integrative literature review
}

\author{
ANTOINETTE MARY FAGE-BUTLER AND MATILDE NISBETH JENSEN
}

Aarhus University, Denmark

\section{Abstract}

Email communication is being integrated relatively slowly into doctor-patient communication. Patients have expressed enthusiasm for the medium, while doctors are generally more reluctant. As existing health communication models have characteristically assumed the copresence of doctor and patient and primarily reflect medical practitioners' perspectives, their suitability in relation to email communication and patients' perspectives warrants further investigation. Following a two-step process and using the methodology of the integrative literature review, 29 articles from 2004-2014 are analysed with the aim of investigating the advantages and disadvantages of the medium of email from the patient's perspective. The findings are compared to the health communication models of biomedicine, patient-centeredness, patient education and patient empowerment to investigate these models' relevance for doctor-patient email communication. Results show that patients identify numerous advantages with email communication, including improved convenience and access, more detailed informational exchanges, greater reflection opportunities, freedom from the medical gaze and the potential to level out power imbalances, as well as a number of primarily medium-related disadvantages. The findings indicate that email can counteract some of the communicative problems associated with biomedicine and suggest the ongoing rel- evance of aspects of the models of patient empowerment, patient-centeredness and patient education for email communication.

Keywords: doctor-patient communication; email; health communication models; integrative literature review; patients' perspectives.

\section{Introduction}

Despite its relatively slow implementation in medical communication compared to other industries (Atherton et al. 2013), email is increasingly being integrated into doctor-patient communication and, given the 'technological turn' in healthcare (Giordano and Benedikter 2011) and the emergence of 'e-health' (e.g. Eysenbach 2001) increased prevalence is predicted (Katzen et al. 2005). However, research indicates that doctors and patients hold divergent opinions about the value of using email when communicating with each other. Patients have widely embraced email communication with their doctors (Anand et al. 2005; McGeady et al. 2008; Klinar et al. 2010), whilst doctors are generally far more circumspect, concerned that communicating with their patients by email will increase workload and the risk of litigation (Weiss 2004; Recupero 2005; Lang and Kiel 2008). Given its present relatively infrequent use (Ye et al. 2010), the integration of email within doctor-patient communication has been termed a 'new frontier' in doctor-patient communication; its empirical qualities and 
theoretical implications are only beginning to be understood (Schiller et al. 2013).

This integrative literature review was motivated by the combination of patients' eagerness, doctors' reticence, the as-yet only nascent understandings of the potential of the medium for doctor-patient communication, as well as the questionable relevance of existing health communication models for computer-mediated health communication. The study has two interrelated aims and follows a two-step procedure. First, as studies of doctor-patient email communication have mainly been carried out from the doctor's perspective (Andreassen 2011), this paper explores positive and negative aspects of email for patients in order to promote 'state of the art' understandings of the potential of email as a medium of doctor-patient communication. Second, the article compares patients' experiences of email communication with their doctors with the communication models of biomedicine, patient-centeredness, patient education and patient empowerment (Beisecker and Beisecker 1993; Lupton 2003; Pollock 2005). In this way, existing models of health communication, whose values and constructs underpin notions of best practice, are interrogated by patients' stated preferences so as to investigate the relevance of existing health communication models for email communication. This approach was inspired by Ye et al.'s (2010) literature review of empirical articles produced in the US between 2000 and 2008, which concluded that not only are there large lacunae in our understanding of the implications of email use in doctor-patient communication, but which also ended with the tantalizing observation that ' $[\mathrm{e}]$-mail also helps address some unmet needs for communication in health care' (Ye et al. 2010: 273).

Our aims are best investigated using the method of integrative literature review, which has theoretical as well as empirical ambitions (Torraco 2005). Moreover, the inclusion of both positive and negative aspects of email communication from the patient perspective provides a nuanced picture which helps support the identification and promotion of best policy and practice, also a goal of integrative literature reviews (Whittemore and Knafl 2005).

\section{Method}

The role of email in patient-provider communication has previously been the topic of literature reviews, of which that of Ye et al. (2010) is the most notable and recent. However, while Ye and her colleagues conducted a systematic review, the present paper, by contrast, relies on the method of the integrative literature review. Unlike a systematic literature review, this approach encompasses both theoretical and empirical articles of a quantitative and qualitative nature. The integrative literature review was chosen because methodologically heterogeneous article types can 'result in a comprehensive portrayal of complex concepts, theories, or health care problems' (Whittemore and Knafl 2005: 548). Due to its empirical, theoretical and applied strengths, the integrative review method has made many significant contributions to qualitative healthrelated research (Whittemore and Knafl 2005).

An integrative literature review includes the following stages: problem identification, literature search, data evaluation, data analysis, presentation and conclusion (Whittemore and Knafl 2005; Pentland et al. 2011). The articles which constitute the data for this review are primarily derived from two databases, PubMed and Scopos. Key search words used to identify relevant articles were 'email'/'e-mail' or 'web messaging'/'web-messaging' used in the title, and 'patient' in the title or abstract. In August 2014, we searched articles published from 2004 to 2014. Three main types of article feature in the data-set: (1) empirical articles that include focus on patients' perspectives on email communication with their doctors, (2) theoretical articles that include discussions of the implications for patients of email communication with their doctors, and (3) literature reviews which draw conclusions about patients' use of email communication with their doctors. Saturation point in compiling the corpus was quite quickly reached, as many duplicates were present across the two databases; these searches were supplemented by a hand-picking approach, whereby the articles' reference lists were checked for other relevant articles. As this review identifies the perspectives of patients, articles were excluded

\section{e是uinoxonlıne}


if they focused on issues such as the medical outcomes and financial benefits associated with doctor-patient email communication, the security of email from an institutional perspective or simply provided information on the numbers of doctors and patients using email as an adjunct to the clinical setting. We did not include textmessaging in our search, as, while equally relevant, it deserves an entire literature review in itself. A total of 29 peer-reviewed empirical and theoretical articles of international provenance from 2004-2014 met the sampling criteria and are presented in Table 1.
The articles were analysed using the qualitative analysis software NVivo (from QSR International Pty Ltd), and analysis was conducted in line with the following procedure. Both authors coded the articles separately for positive and negative aspects of email for patients. Codes were discussed and agreed on before a second separate coding round was commenced, in which all the codes were checked by both coders. The codes that reflected common areas of concern were then grouped to produce overarching themes. These themes are presented in Section 3 , which discusses the findings associated with

Table 1. Data set of 29 peer-reviewed empirical and theoretical articles

\begin{tabular}{|c|c|c|c|}
\hline & Article & Research aim & Method \\
\hline 1 & Albersheim 2010 & $\begin{array}{l}\text { To outline potential misuses and abuses of } \\
\text { doctor-patient email communication, with clin- } \\
\text { ical and ethical implications, providing practical } \\
\text { suggestions }\end{array}$ & Theoretical \\
\hline 2 & Anand et al. 2005 & $\begin{array}{l}\text { To evaluate the content of emails between doc- } \\
\text { tors and parents of patients in paediatric pri- } \\
\text { mary care, and investigate parent attitudes about } \\
\text { email }\end{array}$ & $\begin{array}{l}\text { Quantitative analysis of emails } \\
\text { plus questionnaire for parents }\end{array}$ \\
\hline 3 & Andreassen 2011 & $\begin{array}{l}\text { To explore how an electronic address was inte- } \\
\text { grated into patients' everyday health practice }\end{array}$ & $\begin{array}{l}\text { Qualitative interviews with } \\
\text { families }\end{array}$ \\
\hline 4 & Andreassen et al. 2006 & $\begin{array}{l}\text { To illuminate changes that patients made } \\
\text { possible when they chose to communicate } \\
\text { electronically with their doctor, with focus on } \\
\text { trust }\end{array}$ & $\begin{array}{l}\text { Qualitative interviews with } \\
\text { families }\end{array}$ \\
\hline 5 & Atherton et al. 2009 & $\begin{array}{l}\text { To perform an overview of four reviews of email } \\
\text { use in the healthcare situation }\end{array}$ & Review \\
\hline 6 & Atherton et al. 2013 & $\begin{array}{l}\text { To understand the use of email consultation in } \\
\text { general practice by investigating the experiences } \\
\text { of existing users and views of experts }\end{array}$ & $\begin{array}{l}\text { Qualitative interviews with } \\
\text { patients, GPs and experts on the } \\
\text { use of email in primary care }\end{array}$ \\
\hline 7 & $\begin{array}{l}\text { Bhuvaneswar and Gutheil } \\
2008\end{array}$ & $\begin{array}{l}\text { To describe the use of email in psychotherapy, } \\
\text { using composite case material }\end{array}$ & Theoretical \\
\hline 8 & Car and Sheikh 2004a & $\begin{array}{l}\text { To describe how email is used for communica- } \\
\text { tion in various clinical contexts }\end{array}$ & Review \\
\hline 9 & Car and Sheikh 2004b & $\begin{array}{l}\text { To summarize the evidence describing public and } \\
\text { professional attitudes to using email in health care }\end{array}$ & Review \\
\hline 10 & Collins et al. 2007 & $\begin{array}{l}\text { To reveal the usefulness of an adjunctive role } \\
\text { for email communication between patients and } \\
\text { their providers in the treatment of substance } \\
\text { abuse }\end{array}$ & Qualitative case study \\
\hline 11 & Hansen et al. 2014 & $\begin{array}{l}\text { To investigate the ways in which patients and } \\
\text { general practitioners communicate with each } \\
\text { other by email }\end{array}$ & $\begin{array}{l}\text { Qualitative focus group inter- } \\
\text { views with patients and GPs }\end{array}$ \\
\hline
\end{tabular}


Table 1. (Continued)

12 Hartgers and Jatoi 2010

Houston et al. 2004

Hsiao et al. 2011

Neville et al. 2004
Schiller et al. 2013

Plener et al. 2014

Recupero 2005

Roter et al. 2008

Tjora et al. 2005

Wallwiener et al. 2009

Weiss 2004

Ye et al. 2010
To describe a case in which a diagnosis occurred Case study by means of a patient-initiated photographic electronic communication

To explore the experiences of patients who were early adopters of email communication with their doctors

To evaluate the impact of a secure electronic messaging system in a paediatric clinic

To elucidate patients' interest in and perspectives about email communication

Mixed methods: quantitative online survey and qualitative interviews

Mixed methods: quantitative online survey and qualitative interviews

Quantitative survey sent to patients

To find out what motivates patients to use email, Quantitative survey sent to whether they were satisfied with it and what impacts it may have had on health behaviour

To examine effects of email on the doctorpatient relationship

To examine ethical, legal and social consequences of email in dermatology practice

patients

Theoretical

Theoretical

Review messaging

To evaluate an email service between patients and their doctors

Mixed methods: quantitative and qualitative analysis of email interactions and quantitative survey

To review doctor-patient

Review

email communication literature in

the context of chronic disease and with a specific focus on inflammatory bowel disease

To address relevant law and ethics guidelines and provide guidelines on professional use of email

To explore the extent to which email messages between patients and doctors are patient-centered

To investigate patient preferences about email communication to inform training

To study the experiences of patients who were using a secure electronic communication system

To review literature on secure patient messaging

To review the literature on clinical, financial, Review legal and ethical implications of medical consultation by email

To examine the discrepancy between patients' enthusiasm for email and doctors' greater reticence, with practical suggestions offered

To review research into email content and the perspectives of doctors and patients on email communication
Theoretical

Quantitative coding of email messages

Mixed methods: qualitative focus group interviews and quantitative assessment scores for students' emails

Qualitative interviews with patients

Review

Theoretical

Review 
the article's empirical purpose - namely, to review relevant literature in order to identify reasons for patients' general support for email communication.

\section{Results for aim 1: Reviewing positives and negatives}

In what follows, positive and negative aspects of email communication for patients are presented separately. Note that the numbers in brackets refer to the numbers in Table 1.

\subsection{Positive aspects of doctor-patient email communication for patients}

Our analysis identified the following positive aspects of doctor-patient email communication.

\subsubsection{Access and convenience}

In many of the articles, greater access and extra convenience are underlined as major advantages of email. Email provides flexibility of access for both parties, as emails can be sent and read at a suitable time, outside traditional office hours and where convenient $(2,4-8,11,12,15,16,19-21$, 23-26, 28). Email also supports patients who may not otherwise be able to contact their practitioner easily; it may even be the only tool that makes access possible, for reasons such as geography, weather conditions, disability or social stigma (3-5, 7, 8, 10, 20, 21, 26, 29). Another advantage of having direct access to the doctor is that patients may be able to bypass reception $(6,13)$ and avoid telephone queues $(11)$.

Patients associate the access that email provides with efficiency and time-saving $(1,6,8$, $10,13-15,19,21,26,27)$. Patients also consider email to be time-efficient for doctors, which is helpful for patients who are reluctant to take up doctors' time in the clinic with simple inquiries or questions (20). Email consultation is described as a middle ground between a face-to-face appointment and no appointment at all (5), or 'the next best thing to a personal house call' (17).

Email communication thus facilitates more frequent doctor-patient communication (19), which can reassure patients (3). Patients are relieved that, having sent an email, the responsibility for assessing the illness lies with their doctor, which might be especially relevant in the pediatric setting (3). Increased access to their doctor supported by email can help the doctor-patient relationship to develop as patients receive ongoing feedback and help with decision-making $(10,21)$. It is suggested that just having email access can create 'a sense of a virtual physician who is there with you all the time, albeit asynchronously' (28). Patients' ability to contact the doctor from their home has been linked to a reduction in the need for primary care and for specialist and emergency visits (21).

\subsubsection{More detailed informational exchanges possible}

Email can lead to greater information flow between doctor and patient and vice versa (3, 21, 25). Emails can be as long as need be, and include attachments (8) such as patients' pictures of skin ailments $(11,12)$. Some patients find it useful to let their doctor know what is happening in advance of an appointment $(10,20)$. Email provides an additional medium of communication for patients who might not otherwise get to communicate with their doctors about issues such as new symptoms and the progression or resolution of existing problems $(2,8)$ or who consider themselves too busy to wait at doctors' offices $(15,16,20)$. In addition, regular communication between patient and provider improves the efficiency of face-to-face sessions (25), where little time is available to provide updates on life events (10).

Some studies found not only that email lowered the threshold for contacting the doctor, but also that the content of the exchange changed with the medium shift: patients ask questions electronically that they would not have asked in an ordinary consultation $(4,19,20)$. Email can also be used for follow-up, for instance, after an appointment with a doctor when clarification or additional information may be required $(5,17)$. Email can be especially valuable for patients with chronic diseases $(5,28)$, as more updates and monitoring can improve continuity of care (19).

Email can also be a gateway to better patient learning opportunities, as doctors can attach written sources or provide internet links (2,

\section{e是uinoxonlıne}


$8,11,26)$, which supports informed decisionmaking and patient autonomy (18). Patients cite email supporting the acquisition of knowledge as a positive $(3,11)$. Some patients use email to request the provision of expert information, such as the findings of clinical trials and information on medication (14). Because of the asynchronous nature of the email medium, doctors are also able to consult with colleagues and other professionals to provide more considered responses to patients' emails (8). As such, email might play a valuable role in safety-netting, where clinical decisions regarding diagnosis and ongoing care are made more robust through the integration of heterogeneous information sources, potentially an indirect informational benefit of email for patients.

\subsubsection{Email as documentation}

Email is self-documenting (21) and is therefore a much less transient form of communication than a telephone call or other verbal communication. Its documenting qualities support patients who may find it hard to understand or write down accurately what doctors say in clinics (14). Patients appreciate having a hard copy to print out and refer to $(14,17,26)$, and which can also be cumulative (13). Having a written record aids recall, both for the patient and the doctor $(5,9,14,18,21,27,29)$. It provides patients with a valuable source for later reference (21) which allows them to discuss the content of messages with family or friends to improve understanding (8). Doctors re-reading email messages may be prompted to ask additional questions (1), which may benefit patients.

\subsubsection{Greater reflection}

Email offers patients the opportunity to express thoughts, articulate concerns and ask questions without the tight time constraints of face-to-face encounters $(6,13,15,17,23)$. Doctors, similarly, have more time to contemplate their answers (19), which can also benefit patients. As the time patients take to write an email is self-determined, there is more time to formulate questions and responses as well as to ask forgotten questions or questions that do not get asked if time runs out during the clinical encounter $(11,14,25)$. Some patients find it easier to remember and communicate their health questions and concerns in writing, as they get distracted when speaking to the doctor (4). Email thus supports patients' reflexivity (25), as it requires 'self-assessment, introspection, and acknowledgment of their disorder' (10).

\subsubsection{Emotional support}

Patients report that emails increase their sense of being in touch with and being looked after by their doctor, as doctors become more attentive to therapeutic tasks (8). Patients can be reassured by emails from their doctors when faced, for example, with changes in their condition, resulting in reduced anxiety levels and better quality of life (21). Email provides another form of communication that supports psychiatric patients, allowing them to feel connected with their psychiatrist (5). Emails from psychiatrists can indicate that they care (7), and patients who need psychiatric support may be able to express themselves more freely using email (5). Furthermore, email exchanges can become more personal and clinical visits less emotionally charged on the basis of existing email communication (17). Regular communication between patients and their provider helps combat patient isolation (10).

\subsubsection{Freedom from the medical gaze}

Because email is textual and asynchronous, it can provide patients with a sense of privacy (16) and anonymity $(8,13,22)$. Patients are thus able to express themselves with less inhibition, especially when sensitive or embarrassing issues are at stake $(5,8,10,11,13,16,17,19$, $23,25,26)$. Patients emailing their doctors feel more comfortable asking questions (29), again because email does not involve the normal time constraints of face-to-face encounters (15). One study includes the example of a mother using email and photo attachments to communicate with the doctor about her daughter's eczema, as opposed to bringing her daughter to the clinic. The author notes the mother's motivation to avoid her daughter's condition being 'pathologized', citing it as evidence that email can shield patients from the 'medical gaze' (3). 


\subsubsection{Levelling out of power imbalances} Email can help to address power asymmetries that may otherwise characterise the clinical setting. As noted above, email benefits patients because the doctor-patient encounter is more on their terms, physically and temporally (Section 3.1.1, 'Access and convenience'), and it gives patients freedom from pathologizing practices (Section 3.1.6, 'Freedom from the medical gaze'). Email's potential to level out power asymmetries also reflects the fact that patients using email are in a better position to have their say. One article reported that patients dominated email messages, both in relation to the number of ideas communicated and words used (23), and another presented how patients put forward their own hypotheses for discussion (25). Email also enables patients to be agenda-setters, as emailing most often is patient-initiated $(6,11,12)$. Insofar as email facilitates self-care and greater patient responsibility with regard to health matters $(3,10)$, it offers the possibility of altering the traditional spheres of responsibilities (3). Email supports patients in adopting the role of 'sparring partner' (11), leading to greater patient autonomy and empowerment $(16,18)$ and acting as an antidote to medical paternalism (18).

\subsubsection{Informal language}

The informal and more intimate quality of email communication can strengthen patients' relationship with their doctor (4). The free style of writing is described as an advantage of email communication as it minimises the time taken to write and read messages (8). Moreover, the informality of email also makes it a more egalitarian medium: 'traditional barriers of age, rank, and unfamiliarity tend to dissolve in the informality of electronic communication' (8).

\subsection{Negative aspects of doctor-patient email communication for patients}

In the following sub-sections, the categories associated with patients' negative perspectives on email communication are presented.

\subsubsection{Problems in delivery of feedback}

Email is considered unsuitable for urgent issues because of its asynchronous nature $(1,8,15,17$,
18, 26). Patients recommend quick response times (16), with frustrations about delays being evident (21). Patients may not get a reply (16, $25)$, or may worry about the prospect of not getting one (13); patients may also find waiting for a response to be inconvenient $(20,16,29)$. One participant who used email to set up an appointment felt that the system had not met his expectations because his symptoms had resolved before he received a response to his mail (20).

\subsubsection{Concerns about confidentiality}

Views were divided on the issue of confidentiality. Several studies mention concerns about confidentiality as one of the main negatives $(1,5$, $8,17,19,21,26,27)$, as email leaves a permanent record (1), and an email sent in confidence to a clinic may be forwarded erroneously to the wrong recipient. Concerns about who may be reading or answering patients' emails were expressed in a number of the articles $(14,20,29)$, with some patients concerned that a different doctor than their own may be reading and responding to the query (20). A different picture emerges in other studies. One study shows that patients were evenly divided on confidentiality, with $49 \%$ overall unconcerned and 51\% concerned (15), whilst it is a lesser concern in other papers $(13,25)$.

\subsubsection{Missing human contact}

The medium of email for doctor-patient communication is considered impersonal $(5,7,14)$, with some patients fearing lack of human contact $(14,17)$ as doctors are unable to examine and touch the patient for diagnostic and empathetic purposes $(8,13)$. Many characteristics of live communication are simply not possible in the medium of email, including facial expression and eye contact (1), body language $(1,5,7,8$, $24,25)$ and verbal clues such as tone of voice (1, $5,7,8,14,25)$. The absence of such cues makes it difficult to recognize misunderstandings, and the asynchronous nature of the medium renders it impossible to give instant feedback $(7,9,14$, $18)$. One way in which doctors could compensate for the leaner affordances of the medium of email would be to try to communicate with patients in email as they would in face-to-face or telephone encounters; for example, through the

\section{equinoxonlıne}


use of simple language, by encouraging patients to ask questions, and by summarizing the main points covered (9).

Because of these limitations, email is recommended as an adjunct only to clinical consultations (21). While useful in some therapeutic situations, email has been described as unsuitable for dealing with sensitive matters (1) as it may problematize the communication of compassion and empathy $(7,18)$.

\subsubsection{Trust issues}

Trust is often considered to be a prerequisite for email communication between doctors and patients (4). However, trust can be damaged through negative experiences with email. One patient lost faith in email communication as her doctor had confused her with another patient and responded to her email inquiry on the basis of another patient's diagnosis, prompting the patient to be concerned enough to find another doctor (4). In another study, when doctors replied that the problem was unsuitable for email, patients experienced it as a rejection of their problem (11), which could damage the preexisting relationship. Alternatively, increased communication and levels of trust in doctors can lead to greater risks of medicalization (4). However, although patients know that trust is at stake, many are reasonably confident in trusting email systems, as they implicitly trust their doctors receive their emails (25).

\subsubsection{Content issues}

Email is inappropriate for delivering some messages, such as bad news (24). The way content is delivered can also be problematic. Some patients regard doctors' email responses as being too general, and they want answers that are more detailed, precise, and relevant to their own situation (16). Another content-related disadvantage has to do with the potential for ambiguity in emails, with patients concerned that their emails are misunderstood by their doctors (13), or that they are asked to reformulate questions (16). Similarly, email's removal from real-time, where context and tone are lost $(14,18)$, can lead to misunderstanding $(1,5,7,11$, 24). This may have the deleterious consequence for patients of increased diagnostic errors (7, 8). Similarly, some patients complain about the technical lexis and dense writing style of some doctors (24). A further issue with email content is its manipulability: a message can inadvertently be altered through erroneous deletions, typographic errors and cutting and pasting (18, 21). Grammatical mistakes made by doctors can also be disconcerting for patients (24). Finally, patients are concerned about the fact that they are responsible for filtering information to their doctors in emails, which necessitates competence in sifting between medically relevant and irrelevant information (4).

\section{Results for aim 2: Implications for health communication models}

We now turn to the second aim of our integrative literature review, which is to consider the theoretical implications of the review's empirical findings for the ongoing relevance of existing health communication models. As noted in the introduction, theoretical insights into the implications of electronically mediated communication between doctors and patients are still sparse; the increasing application of electronically mediated doctor-patient communication poses theoretical challenges for existing models of communication intended for the clinical situation (Lang and Kiel 2008; Ye et al. 2010). The comparative approach adopted here makes it possible to identify those aspects of existing health communication paradigms for the clinical situation that may be relevant for doctorpatient email communication. Categories that do not match existing health communication paradigms also help to indicate areas where theoretical development is needed. The models of biomedicine, patient-centeredness, patient empowerment and patient education, which are recognized as the dominant paradigms for the clinical situation (Beisecker and Beisecker 1993; Lupton 2003; Pollock 2005), are now considered within the context of the empirical results of the integrative literature review, which were presented in Section 3. 


\subsection{The positives of email communication}

Biomedicine was originally an approach to diagnostic inquiry (Balint 1969; Engel 1977), rather than a health communication paradigm per se. However, it was used as a default approach to communication. Biomedicine prioritizes a scientific approach in the clinical situation, in which the focus is primarily on what the expert doctor knows rather than on what the patient experiences. In the findings of our review that reflect the 'positives' of email communication with doctors, it would appear that some of the insufficiencies of biomedicine as a paradigm for health communication are counteracted: email manages to circumvent biomedicine's oppressive 'clinical gaze' and enables patients to become agendasetters, in a way that corrects (at least partially) biomedicine's power imbalances, where doctors have institutionally defined power associated with their professional expertise (Foucault 1972: 50).

Many of the advantages of email relate to patient empowerment (e.g. Feste and Anderson 1995). Greater access and convenience mean that email communication is on patients' terms to a far greater degree; patients are liberated to ask new questions in the medium because of the quasi-anonymity it offers; and patients gain in autonomy as doctors' more detailed responses mean that they are better equipped to deal with their condition. Levelling out power imbalances relates in an obvious way to patient empowerment. More informal language can also be empowering for patients.

The advantages of email communication between doctors and patients can also be linked to patient-centeredness, which espouses greater awareness of the patient as a person (Balint 1969; Engel 1977). Email can improve the doctor-patient relationship within the framework of ongoing care as increased access and convenience are reassuring and an indication that the doctor cares. Patients can receive email for emotional support; it can also help patients express their feelings about their illness. This is exemplified in a study by Roter et al. (2008: 85) which concluded that email supports patientcenteredness as patients are more emotionally invested in emails, partly due to the disinhibiting qualities of communicating in the absence of time limits and the fact that patients can communicate from the familiar surroundings of their home. Informal language can also suggest greater immediacy. The value of email for patientcentered communication is also evident in that patients are able to set the agenda. The levelling out of power imbalances is relevant here, as patient-centered communication strives for more equal power relationships. Schiller et al. (2013:64) conclude that patient-centered clinical communication may have similarities with 'ideal physician-patient email communication.

Patient education is a health communication paradigm that prioritizes the encounter as a learning experience for patients (e.g. Kelly 2011). Greater information flow in both directions supports patient education, and doctors are able to provide links and further reading material which is not possible in clinical encounter. Email supports patient education, as it reinforces reflection and recall. As it is self-documenting, patients can use email as a resource for learning.

\subsection{The negatives of email communication}

The categories related to negative aspects of email communication were more difficult to map against existing health communication paradigms traditionally conceived for the clinical situation, as these categories can mainly be explained by reference to the medium itself. Thus, not hearing back at all or hearing back when it is too late relate to the asynchronous nature of email. Worries about confidentiality can be attributable to the opaque recipient of email messages sent to clinics. Concern about missing human contact relates to the asynchronous and textual nature of email. Regarding trust, as Giddens (1990: 17) points out, greater demands are placed on trust when people have to rely on technological systems, or what he calls the 'disembedding of social systems.' The question of responsibility for decisions arises, as does the question of accountability for communication and diagnosis errors. As Recupero (2005: 465) asserts, the medium of email provokes a new set of legal and ethical questions. Patients' critique of the lack of (appropriate) email responses from

\section{e是uinoxonlıne}


doctors also recalls patients' frustrations with biomedical communication (Engel 1977).

\section{Discussion and conclusion}

In line with the empirical aims of our paper, we reviewed relevant literature from 2004-2014 and identified the reasons for patients' broad enthusiasm for and, to a lesser extent, reservations about, using email when communicating with their doctor. As this study presents stateof-the-art findings on patients' experiences of email communication with their doctor, it makes a highly-relevant contribution, particularly given the steady integration of email in doctor-patient communication. It is, however, clear that a literature review which examines doctors' perspectives on email communication with their patients would also be very beneficial, particularly as doctors' concerns about overburdening, reimbursement and the legal implications of what they are sending via email are very likely to affect the information included in emails received by patients. There needs to be sustained empirical focus on this area, as present understanding of email communication in the health setting is still in its early stages and adjustments to the medium will continue to be made by doctors and patients alike, also in the context of evolving rules of engagement. It is also evident that those medium-related aspects of email communication which impoverish its quality need to be understood better and addressed. Further empirical research could reveal how it might be possible to compensate for disadvantages that appear largely to be a function of the medium itself.

We also considered the theoretical implications of our findings for existing clinically-related health communication models. Our findings suggest that aspects of patient empowerment, patient-centeredness and patient education are relevant for email communication between patients and doctors, and that email counteracts some of the communicative problems associated with biomedicine. As email enables patients to become agenda-setters, the review points in the direction of new inquiries into 'patient-doctor communication' (rather than doctor-patient communication). With increasing use of email communication as well as other kinds of internet-related media (such as patient portals, text messaging and patient forums in healthcare), we argue for research that supports the development of theoretical frameworks for computer-mediated health communication, to which this integrative review makes a contribution.

The present study has a number of practical implications, as it provides compelling evidence of the value of increased use of email communication in doctor-patient interactions. Indeed, using email to replace face-to-face options, where possible and where advantageous for patients, could free up time and resources for better clinical consultations. Moreover, as the present study identifies patients' perspectives on email communication with their doctors, it may support the development of policies on email communication that reflect patients' preferences. Finally, given the current lack of awareness of how to identify and integrate patients' perspectives on health communication (Fage-Butler 2013), it would be valuable to consider what lessons from email communication might transfer to the clinical setting.

\section{References}

Albersheim, S. (2010) E-mail communication in paediatrics: Ethical and clinical considerations. Paediatrics and Child Health 15 (3): 163-168.

Anand, S. G., Feldman, M. J., Geller, D. S., Bisbee, A. and Bauchner, H. (2005) A content analysis of e-mail communication between primary care providers and parents. Pediatrics 115 (5): 12831288. http://dx.doi.org/10.1542/peds.2004-1297

Andreassen, H.K. (2011) What does an e-mail address add? Doing health and technology at home. Social Science and Medicine 72 (4): 521-528. http://dx. doi.org/10.1016/j.socscimed.2010.11.026.

Andreassen, H. K., Trondsen, M., Kummervold, P. E., Gammon, D. and Hjortdahl, P. (2006) Patients who use e-mediated communication with their doctor: New constructions of trust in the patient-doctor relationship. Qualitative Health Research 16 (2): 238-248. http://dx.doi.org/10.1177/10497323052 84667

Atherton, C., Car, J. and Meyer, B. (2009) Email for clinical communication between patients/

\section{equinoxonlıne}


caregivers and healthcare professionals. Cochrane Database of Systematic Reviews 3: 1-16. http:// dx.doi.org/10.1002/14651858.CD007978.pub2

Atherton, H., Pappas, Y., Heneghan, C. and Murray, E. (2013) Experiences of using email for general practice consultations: A qualitative study. British Journal of General Practice 63 (616): e760-7.http:// dx.doi.org/10.3399/bjgp13X674440

Balint, E. (1969) The possibilities of patient-centered medicine. Journal of the Royal College of General Practitioners 17 (82): 269-276.

Beisecker, A. E. and Beisecker, T. D. (1993) Using metaphors to characterize doctor-patient relationships: Paternalism versus consumerism. Health Communication 5 (1): 41-58. http://dx.doi.org/ 10.1207/s15327027hc0501_3

Bhuvaneswar, C. G. C. and Gutheil, T. G. T. (2008) E-mail and psychiatry: Some psychotherapeutic and psychoanalytic perspectives. American Journal of Psychotherapy 62 (3): 241-261.

Car, J. and Sheikh, A. (2004a) Email consultations in health care: 2 - acceptability and safe application. British Medical Journal 329: 439-442. http://dx. doi.org/10.1136/bmj.329.7463.439

Car, J. and Sheikh, A. (2004b) Email consultations in health care: 1 - scope and effectiveness. British Medical Journal 329: 435-438. http://dx.doi.org/ 10.1136/bmj.329.7463.435

Collins, G. B., McAllister, M. S. and Ford, D. B. (2007) Patient-provider e-mail communication as an adjunctive tool in addiction medicine. Journal of Addictive Diseases 26 (2): 45-52. http://dx.doi. org/10.1300/J069v26n02_06

Engel, G. L. (1977) The need for a new medical model: A challenge for biomedicine. Science 196 (4286): 129-136. http://dx.doi.org/10.1126/science.847 460

Eysenbach, G. (2001) What is e-health? Journal of Medical Internet Research 3 (2). http://dx.doi.org/ 10.2196/jmir.3.2.e20

Fage-Butler, A. (2013) Including patients' perspectives in patient information leaflets: A polyocular approach. Fachsprache 35 (3/4): 140-154.

Feste, C. and Anderson, R. M. (1995) Empowerment: From philosophy to practice. Patient Education and Counseling 26 (1-3): 139-144. http://dx.doi. org/10.1016/0738-3991(95)00730-N

Foucault, M. (1972) The Archaeology of Knowledge. New York: Pantheon.

Giddens, A. (1990) The Consequences of Modernity. Cambridge: Polity.

Giordano, J. and Benedikter, R. (2011) The shifting architectonics of pain medicine: Toward ethical realignment of scientific, medical and market values for the emerging global community: Groundwork for policy. Pain Medicine 12 (3): 406-414. http://dx.doi.org/10.1111/j.1526-4637. 2011.01055.x

Hansen, C. S., Christensen, K. L. and Ertmann, R. (2014) Patients and general practitioners have different approaches to e-mail consultations. Danish Medical Journal 61 (6): 1-4.

Hartgers, M. and Jatoi, A. (2010) E-Mail and photographs: A case report of a patient-initiated diagnostic tool in the era of electronic communication. Journal of Palliative Medicine 13 (3): 335-337. http://dx.doi.org/10.1089/jpm.2009.0215

Houston, T. K., Sands, D. Z., Jenckes, M. W. and Ford, D. E. (2004) Experiences of patients who were early adopters of electronic communication with their physician: Satisfaction, benefits, and concerns. The American Journal of Managed Care 10 (9): $601-$ 608.

Hsiao, A. L., Bazzy-Asaad, A., Tolomeo, C., Edmonds, D., Belton, B. and Benin, A.L. (2011) Secure web messaging in a pediatric chronic care clinic: A slow takeoff of 'Kids' airmail'. Pediatrics 127 (2): e406e413. http://dx.doi.org/10.1542/peds.2010-1086

Katzen, C., Solan, M. J. and Dicker, A. P. (2005) E-mail and oncology: A survey of radiation oncology patients and their attitudes to a new generation of health communication. Prostate Cancer and Prostatic Diseases 8 (2): 189-193. http://dx.doi. org/10.1038/sj.pcan.4500797

Kelly, R. B. (2011) Patient education. In E. Rakel and D. P. Rakel (eds) Textbook of Family Medicine, 160-165. Eighth edition. Philadelphia, PA: Elsevier Saunders.

Klinar, I., Balazin, A., Basic, M., Plantas, I. and Biskupic, K. (2010) Increased demand for e-mail health consultation service: Analysis of a web survey. Collegicum Antropologicum 34 (2): 481-485.

Lang, K. T. and Kiel, J. M. (2008) An analysis of the utilization of e-mail by physicians and patients. Journal of Healthcare Information Management $22(2): 27-32$.

Luo, J., Logan, C., Long, T. P. and Bercovitch, L. (2009) Cyberdermatoethics I: Ethical, legal, technologic, and clinical aspects of patient-physician e-mail. Clinics in Dermatology 27 (4): 359-366. http://dx. doi.org/10.1016/j.clindermatol.2009.02.008

Lupton, D. (2003) Medicine as Culture: Illness, Disease and the Body in Western Societies. London: Sage.

McGeady, D., Kujala, J. and Ilvonen, K. (2008) The impact of patient-physician web messaging on 
healthcare service provision. International Journal of Medical Informatics 77 (1): 17-23. http://dx.doi. org/10.1016/j.ijmedinf.2006.11.004.

Neville, R. G., Marsden, W., McCowan, C., Pagliari, C., Mullen, H. and Fannin, A. (2004) Email consultations in general practice. Informatics in Primary Care 12 (4): 207-214.

Pentland, D., Forsyth, K., Maciver, D., Walsh, M., Murray, R., Irvine, L. and Sikora, S. (2011) Key characteristics of knowledge transfer and exchange in healthcare: Integrative literature review. Journal of Advanced Nursing 67 (7): 1408-1425. http:// dx.doi.org/10.1111/j.1365-2648.2011.05631.x

Plener, I., Hayward, A. and Saibil, F. (2014) E-mail communication in the management of gastroenterology patients: A review. Canadian Journal of Gastroenterology and Hepatology 28 (3): 161165.

Pollock, K. (2005) Concordance in Medical Consultations. Abingdon: Radcliffe Publishing.

Recupero, P. R. (2005) E-mail and the psychiatristpatient relationship. Journal of the American Academy of Psychiatry and the Law 33 (4): 465475.

Roter, D., Larson, S., Sands, D. Z., Ford, D. E. and Houston, T. (2008) Can e-mail messages between patients and physicians be patient-centered? Health Communication 23 (1): 80-86. http://dx. doi.org/10.1080/10410230701807295

Schiller, J. H., Christner, J. G., Stansfield, R. B., Watnick, C. S. and Mullan, P. B. (2013) What parents want from emails with their pediatrician: Implications for teaching communication skills. Patient Education and Counseling 92 (1) 61-66. http://dx.doi. org/10.1016/j.pec.2013.02.012

Tjora, A., Tran, T. and Faxvaag, A. (2005) Privacy vs usability: A qualitative exploration of patients' experiences with secure internet communication with their general practitioner. Journal of Medical Internet Research 7 (2): e15. http://dx.doi.org/10. 2196/jmir.7.2.e15

Torraco, R. J. (2005). Writing integrative literature reviews: Guidelines and examples. Human Resource Development Review 4 (3): 356-367. http:// dx.doi.org/10.1177/1534484305278283

Wallwiener, M., Wallwiener, C. W., Kansy, J. K., Seeger, H. and Rajab, T. K. (2009) Impact of electronic messaging on the patient-physician inter- action. Journal of Telemedicine and Telecare 15 (5): 243-250. http://dx.doi.org/10.1258/jtt.2009.09 0111

Weiss, N. (2004) E-mail consultation: Clinical, financial, legal, and ethical implications. Surgical Neurology 61 (5):455-459. http://dx.doi.org/10.1016/j. surneu.2003.09.029

Whittemore, R. and Knafl, K. (2005) The integrative review: Updated methodology. Journal of Advanced Nursing 52 (5):546-553. http://dx.doi.org/ 10.1111/j.1365-2648.2005.03621.x

Wong, R. K. M., Tan, J. S. M. and Drossman, D. A. (2010) Here's my phone number, don't call me: Physician accessibility in the cell phone and e-mail era. Digestive Diseases and Sciences 55 (3): 662-667. http://dx.doi.org/10.1007/s10620-009-1089-5

Ye, J., Rust, G., Fry-Johnson, Y. and Strothers, H. (2010) E-mail in patient-provider communication: A systematic review. Patient Education and Counseling 80 (2): 266-273. http://dx.doi.org/ 10.1016/j.pec.2009.09.038

Antoinette Mary Fage-Butler gained her PhD from Aarhus University, where she is currently Assistant Professor, and is a member of both the Knowledge Communication Research Group and the Health Communication Project Group. Her main research interests are within online health communication (doctor-patient and patient-patient), ethical aspects of health communication, and poststructuralist approaches to discourse and genre. Address for correspondence: Department of Business Communication, Aarhus University, 8000 Aarhus C, Denmark. Email: fage-butler@bcom.au.dk

Matilde Nisbeth Jensen gained her cotutelle PhD from Macquarie University and Aarhus University. She currently holds an Assistant Professorship at Aarhus University, where she is a member of the Research Group for Translation and Interpreting and the Health Communication Project Group. Her research interests include medical translation, computer-mediated health communication, health literacy and patient-patient communication. Address for correspondence: Department of Business Communication, Aarhus University, 8000 Aarhus C, Denmark. Email: matnj@bcom.au.dk 